MATEC Web of Conferences 11,01024 (2014)

DOI: $10.1051 /$ matecconf / 20141101024

(C) Owned by the authors, published by EDP Sciences, 2014

\title{
Buckling of Functionally Graded Nanobeams Based on the Nonlocal New First-Order Shear Deformation Beam Theory
}

\author{
HOUARI. M.S.A ${ }^{1,2}$, BOUSAHLA. A.A ${ }^{2}$, BESSAIM. A ${ }^{1,2}$, ADDA BEDIA. E.A ${ }^{2}$ et TOUNSI. A ${ }^{2}$ \\ ${ }^{1}$ Université de Mascara, Département des Sciences \& Technique, Mascara, Algérie \\ ${ }^{2}$ Université de Sidi Bel Abbes, Laboratoire des Matériaux et Hydrologie, Sidi Bel Abbes, Algérie
}

\begin{abstract}
In this work, the size-dependent buckling behavior of functionally graded (FG) nanobeams is investigated on the basis of the nonlocal continuum model. The material properties of FG nanobeams are assumed to vary through the thickness according to the power law. In addition, Poisson's ratio is assumed constant in the current model. The nanobeams is modelled according to the new first order shear beam theory with small deformation and the equilibrium equations are derived using the Hamilton's principle. The Naviertype solution is developed for simply-supported boundary conditions, and exact formulas are proposed for the buckling load. The effects of nonlocal parameter, aspect ratio, various material compositions on the stability responses of the FG nanobeams are discussed.
\end{abstract}

Keywords. Buckling, nonlocal elasticity theory, functionally graded nanobeams.

\section{Introduction}

Functionally graded materials (FGMs) are made from a mixture of two materials to achieve a composition that provides certain functionality. Recently, the application of FGMs has broadly been spread in micro-and nanoscale devices and systems such as thin films [1]. This is achieved by gradually varying the volume fraction of the constituent materials. During the past decade, FGMs have been widely used in various aspects of engineering sciences, such as aerospace, nuclear, civil, automotive, optical, biomechanical, electronic, chemical, mechanical, and shipbuilding industries. With the development of the material technology, FGMs have been employed in micro/nano-electro-mechanical system (MEMS/NEMS) [2]. Stability studies of nanostructure made of functionally graded materials are of great significance for estimating the performance of FG nanobeams. In recent years, various investigations have been carried out to study the buckling [3], bending analysis [4,6], and free vibration [5] of the FG nanobeams in which the classical continuum mechanics have been employed.

This paper focuses on the buckling of the FG nanobeam based on the nonlocal refined first order shear beam theory. The material properties of FG nanobeams are assumed to vary through the thickness according to the power law. In addition, Poisson's ratio is assumed constant in the current model. The nanobeam is modelled according to the new Timoshenko beam theory with small deformation and the equilibrium equations are derived using the Hamilton's principle. The Navier-type solution is developed for simply-supported boundary conditions, and exact formulas are proposed for the buckling load. The effects of nonlocal parameter, aspect ratio, various material compositions on the stability responses of the FG nanobeams are discussed.

\section{Theoretical formulations}

\subsection{Basic assumptions of the refined first shear deformation beam theory}

The displacement field of the proposed theory is chosen based on the following assumptions:

(i) The displacements are small in comparison with the plate thickness and, therefore, strains involved are infinitesimal.

(ii) The transverse displacement $w$ components are functions of coordinates $\mathrm{x}, \mathrm{y}$ only.

$w(x, y, z)=w(x, y)$ 
(iii) The transverse normal stress $\sigma_{z}$ is negligible in comparison with in-plane stresses $\sigma_{x}$ and $\sigma_{y}$.

(iv) The displacements $u$ in $\mathrm{x}$-direction consist of extension and bending components.

$U=u_{0}+u_{b}$

The bending components $\left(u_{b}\right)$ and $\left(v_{b}\right)$ are assumed to be similar to the displacements given by the classical plate theory. Therefore, the expression for $\left(u_{b}\right)$ and $\left(v_{b}\right)$ can be given as

$u_{b}=-z \frac{\partial \phi}{\partial x}$

\subsection{Kinematics and constitutive equations based} on the nonlocal elasticity theory

Based on the assumptions made in the preceding section, the displacement field can be obtained using Eqs. $(1,3)$ as

$$
\begin{gathered}
u(x, y, z)=u_{0}(x, y)-z \frac{\partial \phi}{\partial x} \\
w(x, y, z)=w(x, y)
\end{gathered}
$$

The strains associated with the displacements in Eq. (4) are

$$
\begin{gathered}
\varepsilon_{x}=\varepsilon_{x}^{0}+z k_{x} \\
\gamma_{x z}=\gamma_{x z}
\end{gathered}
$$

Where

$$
\varepsilon_{x}^{0}=\frac{\partial u_{0}}{\partial x}, k_{x}=-\frac{\partial^{2} \phi}{\partial x^{2}}, \gamma_{x z}=\frac{\partial w}{\partial x}-\frac{\partial \phi}{\partial x}
$$

\subsection{Constitutive relations}

The FG microbeam is is made of two different Materials and the effective material properties (Young modulus) of the FG nanobeam vary continuously in the thickness direction (in the $z$ direction). According to the rule of mixture, the effective material properties $E(z)$ can be expressed as:

$E(z)=E_{1}+\left(E_{2}-E_{1}\right)\left(\frac{2 z+h}{2}\right)^{k}$

It is easily seen that $E(z)=E_{2}$, when $z=+h / 2$, and $E(z)=E_{1}, \quad$ when $\quad z=-h / 2$, $v_{1}=v_{2}=v=0.3$. $\boldsymbol{k}$ represent the volume fraction exponent which takes values greater than or equal to zero. The above powerlaw assumption reflects a simple rule of mixtures used to obtain the effective properties of the $E_{1}$ and $E_{2}$ material of FG nanobeams.

Response of materials at the nanoscale is different from those of their bulk counterparts. Nonlocal elasticity is first considered by Eringen [7]. He assumed that the stress at a reference point is a functional of the strain field at every point of the continuum. Eringen [7] proposed a differential form of the nonlocal constitutive relation as

$\sigma_{x}-\mu \frac{d^{2} \sigma_{x}}{d x^{2}}=Q_{11} \varepsilon_{x}$

$\tau_{x z}-\mu \frac{d^{2} \tau_{x z}}{d x^{2}}=Q_{55} \gamma_{x z}$

Where $Q_{11}=E$ and $Q_{55}=G$ are the elastic modulus and shear modulus of the nanobeam, respectively; $\boldsymbol{\mu}=\left(\boldsymbol{e}_{0} \boldsymbol{a}\right)^{2}$ is the nonlocal parameter, $\boldsymbol{e}_{0}$ is a constant appropriate to each material and $\boldsymbol{a}$ is an internal characteristic length.

\subsection{Governing equations}

In order to obtain the equations of motion, the principal of the Hamilton's principle is required. The principle can be stated in analytical form as

$\delta \int_{0}^{T}(U+V) d t=0$

Where $\delta U$ is the virtual variation of the strain energy; $\delta V$ is the virtual variation of the potential energy. The variation of the strain energy of the beam can be stated as

$$
\begin{aligned}
\delta U & =\int_{0}^{L} \int_{A}\left(\sigma_{x} \delta \varepsilon_{x}+\tau_{x z} \delta \gamma_{x z}\right) d A d x \\
& =\int_{0}^{L}\left(N_{x} \frac{\partial \delta u}{\partial x}-M_{x} \frac{\partial^{2} \delta \phi}{\partial x^{2}}+Q_{x z}\left(\frac{\partial \delta w}{\partial x}-\frac{\partial \delta \phi}{\partial x}\right)\right) d x
\end{aligned}
$$

Where $(N),(M)$ and $(Q)$ are the stress resultants defined as

$$
\left(N_{x}, M_{x}\right)=\int_{A}(1, z) \sigma_{x} d A \text { and } Q_{x z}=\int_{A} k_{s} \tau_{x z} d A
$$

Where $k_{s}$ is the shear correction factor.

The variation of the potential energy by the applied loads can be written as

$\delta V=\int_{0}^{L} q \delta w d x+\int_{0}^{L} N_{0} \frac{d w}{d x} \frac{d \delta w}{d x} d x$ 
Where $(q)$ and $\left(\boldsymbol{N}_{0}\right)$ are the transverse and axial loads, respectively.

Substituting the expressions for $(\delta U)$ and $(\delta V)$ from Eqs. (10), (12) and (13) into Eq. (9) and integrating by parts, and collecting the coefficients of $\left(\delta u_{0}\right),(\delta w)$, and $(\delta \phi)$, the following equations of motion of the proposed beam theory are obtained

$\delta u: \frac{\partial N_{x}}{\partial x}=0$

$\boldsymbol{\delta} \phi: \frac{\partial^{2} \boldsymbol{M}_{\boldsymbol{x}}}{\partial \boldsymbol{x}^{2}}-\frac{\partial \boldsymbol{Q}_{x z}}{\partial \boldsymbol{x}}=0$

$\boldsymbol{\delta} \boldsymbol{w}: \frac{\partial \boldsymbol{Q}_{x z}}{\partial \boldsymbol{x}}+\boldsymbol{q}-\boldsymbol{N}_{c r} \frac{\boldsymbol{d}^{2} \boldsymbol{w}}{\boldsymbol{d x ^ { 2 }}}=0$

By substituting Eq. (6) into Eq. (8) and the subsequent results into Eq. (11), the stress resultants are obtained as

$$
\begin{aligned}
& N-\mu \frac{d^{2} N}{d x^{2}}=A_{11} \frac{d u_{0}}{d x}-B_{11} \frac{d^{2} \phi}{d x^{2}} \\
& M-\mu \frac{d^{2} M}{d x^{2}}=B_{11} \frac{d u_{0}}{d x}-D_{11} \frac{d^{2} \phi}{d x^{2}} \\
& Q-\mu \frac{d^{2} Q}{d x^{2}}=A_{55} \frac{d w_{s}}{d x}
\end{aligned}
$$

Where

$$
\left(A_{11}, B_{11}, D_{11}\right)=\int_{A} Q_{11}\left(1, z, z^{2}\right) d A, A_{s}=\int_{A} k_{s} Q_{55} d A
$$

By substituting Eq. (14) into Eq. (13), the nonlocal equations of motion can be expressed in terms of displacements $\left(u_{0}, w, \phi\right)$ as

$A_{11} \frac{d^{2} u_{0}}{d x^{2}}-B_{11} \frac{d^{3} \phi}{d x^{3}}=0$

$B_{11} \frac{d^{3} u_{0}}{d x^{3}}-D_{11} \frac{d^{4} \phi}{d x^{4}}-A_{55} \frac{d^{2} w_{s}}{d x^{2}}=0$

$A_{55} \frac{d^{2} w_{s}}{d x^{2}}+q-\mu \frac{d^{2} q}{d x^{2}}-N_{c r}\left(\frac{d^{2} w}{d x^{2}}-\mu \frac{d^{4} w}{d x^{4}}\right)=0$

\section{Analytical Solutions for critical buckling of simply supported FG nanobeam}

The above equations of motion are analytically solved for buckling problems. The Navier solution procedure is used to determine the analytical solutions for a simply supported FG nanobeam. The solution is assumed to be of the form

$$
\left\{\begin{array}{l}
u_{0} \\
w_{b} \\
w_{s}
\end{array}\right\}=\sum_{m=1}^{\infty}\left\{\begin{array}{l}
U_{n} \cos (\alpha x) \\
W_{b n} \sin (\alpha x) \\
W_{s n} \sin (\alpha x)
\end{array}\right\}
$$

Where $\left(U_{n}\right),\left(W_{b n}\right)$, and $\left(W_{s n}\right)$ are arbitrary parameters to be determined, and $\alpha=n \pi / L$.

Substituting the expansions of $\boldsymbol{u}_{0}, \boldsymbol{w}_{\boldsymbol{b}}, \boldsymbol{w}_{s}$, and $\boldsymbol{q}$ from Eqs. (17) into Eq. (16), the analytical solutions can be obtained from the following equations

$$
\left(\left[\begin{array}{ccc}
S_{11} & S_{12} & 0 \\
S_{12} & S_{22}-k & 0 \\
0 & 0 & S_{33}-k
\end{array}\right]-\right)\left\{\begin{array}{l}
U_{n} \\
\Phi_{n} \\
W_{n}
\end{array}\right\}=\left\{\begin{array}{l}
0 \\
0 \\
0
\end{array}\right\}
$$

Where

$$
\begin{aligned}
& S_{11}=A_{11} \alpha^{2}, S_{12}=-B_{11} \alpha^{3}, \quad S_{22}=D_{11} \alpha^{4}, \\
& S_{33}=A_{55} \alpha^{2}, \boldsymbol{k}=\lambda N_{c r} \boldsymbol{\alpha}^{2}, \lambda=1+\mu \alpha^{2}
\end{aligned}
$$

\section{Numerical results}

For the purpose of verification, the present model is used to find the first critical buckling load for a simply supported beam made up of a homogenous material where the nonlocal effect is taken into consideration and compare the results with those of Simsek [8]. The nondimensional buckling load defined is defined as:

$$
N_{c r}=\overline{N_{c r}} \frac{L}{E I}
$$

The nondimensional critical buckling load $\overline{N_{c r}}$ of a simply supported beam are presented in Tables 1 for various values of thickness ratio $\mathrm{L} / \mathrm{h}$ and nonlocal parameter $\mu$. The nonlocal parameters $\mu=\left(e_{0} a\right)^{2}$ are taken as $0,1,2,3$, and $4 \mathrm{~nm}^{2}$. These values are taken because $e_{0} a$ should be smaller than $2 \mathrm{~nm}$ for carbon nanotubes as described by Wang and Wang [9].

Table 1 shows that the present buckling loads agree very well with the solutions of Simsek [8] and the solutions of Aydogdu $[10,11]$ for first order shear deformation beam theory.

Table 1. Dimensionless critical buckling load, $\overline{N_{c r}}$ of the homogenous nanobeam.

\begin{tabular}{|l|l|c|c|c|}
\hline$L / h$ & $\mu$ & Aydogdu [27] & Simsek [27] & Present \\
\hline \multirow{4}{*}{10} & 0 & 9.6227 & 9.6226 & 9.6226 \\
\cline { 2 - 5 } & 1 & 8.7583 & 8.7582 & 8.7582 \\
\cline { 2 - 5 } & 2 & 8.0364 & 8.0363 & 8.0363 \\
\cline { 2 - 5 } & 3 & 7.4244 & 7.4244 & 7.4244 \\
\cline { 2 - 5 } & 4 & 6.8990 & 6.8990 & 6.8990 \\
\hline 20 & 0 & 9.8067 & 9.8066 & 9.8066 \\
\cline { 2 - 5 } & 1 & 9.5705 & 9.5705 & 9.5705 \\
\cline { 2 - 5 } & 2 & 9.3455 & 9.3455 & 9.3455 \\
\cline { 2 - 5 } & 3 & 9.1308 & 9.1308 & 9.1308 \\
\cline { 2 - 5 } & 4 & 8.9258 & 8.9258 & 8.9258 \\
\hline 50 & 0 & 9.8595 & 9.8594 & 9.8594 \\
\cline { 2 - 5 } & 1 & 9.8207 & 9.8207 & 9.8207 \\
\cline { 2 - 5 } & 2 & 9.7822 & 9.7822 & 9.7822 \\
\cline { 2 - 5 } & 3 & 9.7440 & 9.7440 & 9.7440 \\
\cline { 2 - 5 } & 4 & 9.7062 & 9.7062 & 9.7062 \\
\hline
\end{tabular}


To investigate the significance of using functionally graded materials on the buckling of nanobeams, the FG nanobeams have the following material properties: $E_{1}=1 T P a, E_{2}=0.25 T P a, v_{1}=v_{2}=0.3$ and where they change across the beam's thickness according to a power law as presented earlier. The shear correction factor is taken $k_{s}=5 / 6$.

Table 2 shows the nondimensional critical buckling load $\overline{N_{c r}}$ of a simply supported nano FGM beam. The nonlocal parameter $e_{0} a$ varies from 0 to $2 \mathrm{~nm}$ and the material distribution parameter $k$ varies from 0 to 10 . The obtained results are compared with those reported by [Simsek] based on nonlocal Timoshenko beam theory. It can be seen that the results of present theory are in excellent agreement with those predicted by [Simsek] for all values of small scale coefficient, material distribution parameter $k$, and length-to-depth ratio of beams.

Table 2. Dimensionless critical buckling load, $\left(P_{c r}=\overline{P_{c r}} L^{2} /\left(E_{1} I\right)\right)$ of the FG nanobeam.

\begin{tabular}{|c|c|c|c|c|c|c|c|c|c|c|c|}
\hline \multirow{3}{*}{$4 / 4$} & \multirow{3}{*}{$k$} & \multicolumn{10}{|c|}{ Noclocal parameter, $e 0 a(\mathrm{~nm})$} \\
\hline & & \multicolumn{2}{|c|}{0} & \multicolumn{2}{|c|}{0.5} & \multicolumn{2}{|c|}{1} & \multicolumn{2}{|c|}{15} & \multicolumn{2}{|c|}{2} \\
\hline & & $\begin{array}{l}\text { FSDT } \\
{[27]}\end{array}$ & Present & $\begin{array}{l}\text { FSDT } \\
{[27]}\end{array}$ & Present & $\begin{array}{l}\text { FSDT } \\
{[27]}\end{array}$ & Present & $\begin{array}{l}\text { FSDT } \\
{[27]}\end{array}$ & Present & FSDT [27] & Present \\
\hline \multirow{5}{*}{10} & 0 & 2.4056 & 2.4056 & 2.3477 & 2.3477 & 2.1895 & 2.1895 & 1.9685 & 1.9685 & 1.7247 & 1.7247 \\
\hline & 0.3 & 3.9921 & 3.9921 & 3.8959 & 3.8959 & 3.6335 & 3.6335 & 3.2667 & 3.2667 & 28621 & 2.8621 \\
\hline & 1 & 5.3084 & 53084 & 5.1805 & 5.1805 & 4.8315 & 4.8315 & 4.3437 & 4.3437 & 3.8059 & 3.8059 \\
\hline & 3 & 6.6720 & 6.6720 & 6.5113 & 6.5113 & 6.0727 & 6.0727 & 5.4596 & 5.4596 & 4.7835 & 4.7835 \\
\hline & 10 & 8.1289 & 8.1289 & 79332 & 7.9332 & 7.3987 & 7.3987 & 6.6518 & 6.6518 & 5.8281 & 5.8281 \\
\hline \multirow{5}{*}{30} & 0 & 2.4603 & 2.4603 & 2.4536 & 2.4536 & 2.4336 & 2.4336 & 2.4011 & 2.4011 & 23570 & 23570 \\
\hline & 0.3 & 4.0811 & 4.0811 & 4.0699 & 4.0699 & 4.0368 & 4.0368 & 3.9828 & 3.9828 & 3.9096 & 3.9096 \\
\hline & 1 & 5.4146 & 5.4146 & 5.3998 & 53998 & 5.3559 & 5.3559 & 5.2843 & 5.2843 & 5.1871 & 5.1871 \\
\hline & 3 & 6.8011 & 6.8011 & 6.7825 & 6.7825 & 6.7273 & 6.7273 & 6.6373 & 6.6373 & 6.5153 & 6.5153 \\
\hline & 10 & 82962 & 82962 & 8.2735 & 8.2735 & 8.2062 & 8.2062 & 8.0964 & 8.0964 & 79476 & 79476 \\
\hline
\end{tabular}

The variation of buckling responses of FG nanobeam with the aspect ratio is presented in Figure 1 for local and non local case with ( $\left.k=1, e_{0} a=1\right)$ and the aspect ratio varies from $\mathrm{L} / \mathrm{h}=5$ to $\mathrm{L} / \mathrm{h}=50$. The critical buckling load predicted by the local (classical) theory is larger than those of the nonlocal results due to the small scale effects. The nonlocal effect decreases the buckling loads especially at high values of nonlocal parameter.

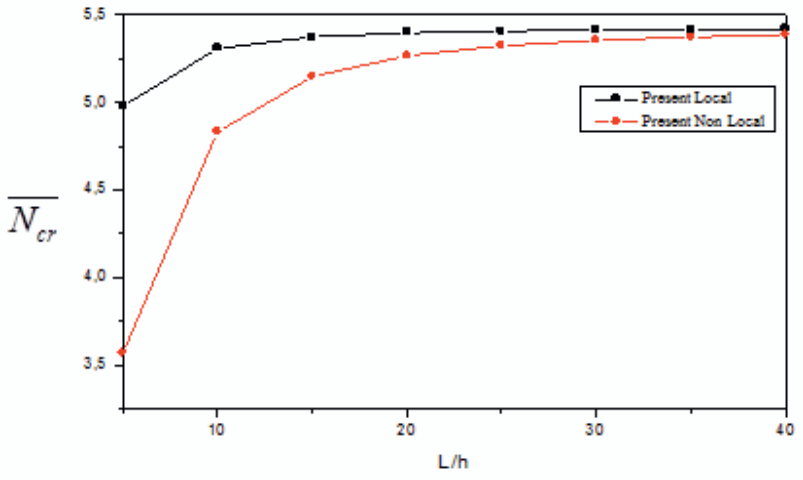

Fig. 1. Effect of the aspect ratio on the dimensionless buckling load for $k=1, \mathrm{e}_{0} \mathrm{a}=1 \mathrm{~nm}$.

The effect of the nonlocal parameter on buckling responses of FG nanobeam is presented in Fig. 2 using present model $(k=1)$ and the aspect ratio varies from $\mathrm{L} / \mathrm{h}=10$ to $\mathrm{L} / \mathrm{h}=50$. According to this figure, the responses of critical buckling load vary nonlinearly with the nonlocal parameter. From this conclusion obtained above, the critical buckling load decreases as the nonlocal parameter increases.

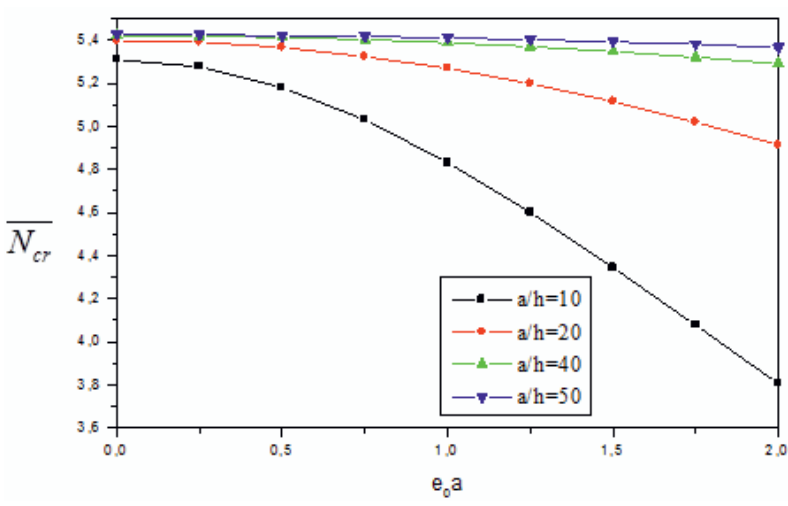

Fig. 2. Effect of the nonlocal parameter on the dimensionless buckling load for $k=1$.

\section{Conclusions}

A simple refined first order shear deformation beams theory was presented for critical buckling of FG nanobeams. Equations of motion are derived from Hamilton's principle. Analytical solutions are obtained for simply supported beams. The effects of nonlocal parameter, aspect ratio, various material compositions on the critical buckling responses of the FG nanobeam are presented and discussed. Numerical results show that the critical buckling load decreases as the nonlocal parameter increases. 


\section{References}

1. Fu Y, Du H, Zhang S. Functionally graded TiN/TiNi shape memory alloy films. Mater Lett 2003; 57(20): 2995-9.

2. Lee Z, Ophus C, Fischer LM, Nelson-Fitzpatrick N, Westra KL, Evoy S, et al. Metallic NEMS components fabricated from nanocomposite Al-Mo films. Nanotechnology 2006; 17(12): 3063-70.

3. A. Heydari, Buckling of functionally graded beams with rectangular and annular sections subjected to axial compression. Int $\mathrm{J}$ Advanced Design and Manufacturing Technology, 2011 5(01): 25-31.

4. Kadoli R., Akhtar K., Ganesan N., Static analysis of functionally graded beams using higher order shear deformation theory. Applied Mathematical Modelling, 32(12), 2509-2525, 2008.

5. Aydogdu M., Taskin V., Free vibration analysis of functionally graded beams with simply supported edges. Materials \& Design, 28(5), 1651-1656, 2007.

6. Benatta M.A., Mechab I., Tounsi A., Adda Bedia E.A., Static analysis of functionally graded short beams including warping and shear deformation effects. Computational Materials Science, 44(2), 765-773, 2008.

7. Eringen AC. On differential equations of nonlocal elasticity and solutions of screw dislocation and surface waves. J Appl Phys 1983;54:4703-10.

8. M. Simsek, H.H. Yurtcu. Analytical solutions for bending and buckling of functionally graded nanobeams based on the nonlocal Timoshenko beam theory. Composite Structures 97 (2013) 378-386.

9. Wang Q. Wave propagation in carbon nanotubes via nonlocal continuum mechanics. J Appl Phys 2005;98:124301.

10. Aydogdu M. A general nonlocal beam theory: its application to nanobeams bending buckling and vibration. Physica E 2009;41:1651-5.

11. Wang, C. M., Zhang, Y. Y., \& He, X. Q. (2007). Vibration of nonlocal Timoshenko beams. Nanatechnology, 18, 105401. 